\title{
Orthogonal transformations and
}

\section{improperly posed problems}

\section{L.S. Jennings}

This thesis examines the application of orthogonal transformations, namely Householder transformations (elementary Hermitians) and plane rotations, to the solution of certain problems which fall into the class of improperly posed problems. Because of the numerical difficulties associated with this class of problems, it is appropriate that orthogonal transformations, with their superior stability properties, should be used to solve them. A brief discussion of some of these problems is presented in the introduction.

A summary of Householder transformations and plane rotations is presented in Chapter 2, together with two orthogonal factorizations of matrices, the $Q-U$ factorization (unitary triangularization) and the singular value decomposition. These are used extensively in the thesis. The concept of a generalized inverse solution to a matrix equation is discussed together with methods for realizing such a solution. In Chapter 3 a direct error analysis for the solution of linear least squares problems using the $Q-U$ factorization shows an interesting result. The error analysis for an underdetermined system is also included.

A new method for the solution of separable elliptic partial differential equations on non-rectangular regions is introduced in Chapter 4. The solution of non-square eigen problems (in an appropriate sense), results from the consideration of finding the free oscillation modes of a fluid in an arbitrarily shaped container. Two new methods are presented for solving the non-square eigen problem. The techniques for solving

Received 5 January 1973. Thesis submitted to the Australian National University, January 1973. Degree approved, June 1973. Supervisors: Dr M.R. Osborne, Dr R.S. Anderssen, Dr K.E. Atkinson. 
Fredholm integral equations of the first kind are summarized in Chapter 5 , and formulated as improperly posed quadratic programing problems. Methods for improperly posed quadratic programming problems are discussed in Chapter 6, where orthogonal transformations are shown to produce certain efficiencies of time and accuracy. 